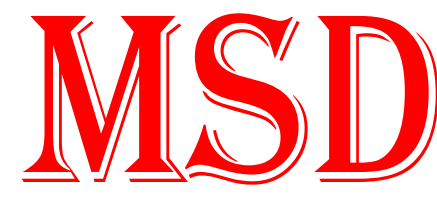

Medical Science and Discovery ISSN: 2148-6832

\section{The clinical, laboratory and prognostic characteristics of haemorrhagic stroke cases related to COVID-19 infection}

\author{
Sibel Üstün Özek ${ }^{1 *}$, Canan Emir $^{1}$ \\ 1 University of Health Sciences, Prof. Dr. Cemil Taşçıŏlu City Hospital, Department of Neurology, Istanbul, TR \\ * Corresponding Author: Sibel Üstün Özek E-mail: sibelustun@hotmail.com
}

\section{ABSTRACT}

Objective: Although ischemic and thrombotic vascular processes are more widely reported in COVID-19, the ratio of haemorrhagic cerebrovascular disease is lower. However, it needs to be evaluated because the mortality rate is higher in haemorrhages, and they may appear iatrogenically.

Material and Methods: Patients observed at the Prof. Dr. Cemil Taşçığlu City Hospital between March 11th, 2020, and March 11th, 2021, were included in the study. Cases diagnosed as consecutive full intracerebral haemorrhage and concomitant with COVID19 were observed during the study period. This study is a cross-sectional, retrospective, and observational study.

Results: Within the 1 -year period, 11 patients ( 7 men and 4 women) with a mean age of $64.45 \pm 18.68$ years related to COVID-19 were recorded. Risk factors were high blood pressure at a frequency of $64 \%$, diabetes mellitus at $45 \%$, and the use of antiaggregants/anticoagulants at $36 \%$. The ratio of male patients was $64 \%(n=7)$. The location of haemorrhage was intraparenchymal in $91 \%(n=10)$, and subdural in $9 \%(n=1)$. The mortality rate was $64 \%$.

Conclusion: Neurologic findings that develop, especially in noncooperating and prone patients in wards and intensive care units, must be observed carefully. Caution must be exercised in prophylactic antiaggregant and anticoagulant treatment, especially in highrisk patients. Intracranial haemorrhages are important due to high mortality.

Keywords: COVID-19; intracranial haemorrhage; haemorrhagic stroke; anticoagulant and antiaggregant treatment; mortality

\section{INTRODUCTION}

The correlation between the development of secondary neurologic symptoms in coronavirus disease 2019 (COVID-19) infection and unfavourable outcomes has been emphasised. Parameters such as increased coagulation tests and D-dimer are correlated with increased coagulopathy (1). An increased risk of haemorrhage along with a thrombosis risk in these patients has been emphasised (2). When evaluating the literature, it is seen that the incidence of haemorrhagic stroke is lower compared with that of ischemic stroke (3). It was proved that COVID-19 caused a thrombosis tendency, and treatment protocols were formed accordingly. There exists a tendency to increase antiaggregant and anticoagulant treatment in high-risk patients (4). However, the risk of the development of haemorrhage should also be considered. The comorbidities in the patient group, and the antiaggregant and anticoagulant treatments administered in treatment, may cause a tendency towards haemorrhage. Although the clinical findings and treatment approaches correlated with COVID-19-related thrombosis are well established, data relating to haemorrhage are limited.

We evaluated the risk factors, concomitant diseases, haematologic values, and administered treatments of patients diagnosed as having and treated for COVID-19, in whom haemorrhage was found as a result of a neurology consultation. We researched haemorrhage distribution rates and mortality based on imaging findings. We planned this study because we thought it was important to draw attention to data on haemorrhages and identify their causes. 


\section{MATERIAL and METHODS}

This is a single-centre, retrospective, cross-sectional, observational study. Prof. Dr. Cemil Taşçığlu City Hospital is a tertiary multidisciplinary hospital, the emergency room of which serves 500,000-550,000 patients every year. At our hospital, approximately 400 patients present to the emergency room pandemic area daily for suspected COVID-19 infection, and around 40 patients are diagnosed as having COVID-19. According to their clinical presentation and findings, outpatient or inpatient treatment is decided for these patients.

Between March 11th, 2020, and March 11th, 2021, 8500 patients with COVID-19 were found to be receiving treatment as inpatients. From among these patients, the clinical and laboratory data for those receiving treatment due to COVID19 and were diagnosed as having haemorrhagic stroke based on the clinical picture and computed tomography (CT) scans, and patients with haemorrhagic cerebrovascular disease who were admitted to the hospital during the same period for causes unrelated to COVID-19 and evaluated by the neurology clinic were examined for the study. The patients' demographic data, existing diseases, neurologic examinations at admission, electrocardiogram (ECG), routine blood analyses [lymphocytes, leukocytes, platelets (PLT), Creactive protein (CRP), fibrinogen, D-dimer, procalcitonin, activated partial thromboplastin time (aPTT)], and cranial imaging (CT) were recorded. The medication the patients used, especially their antiaggregant and anticoagulant use, was recorded. Concomitant comorbidities were recorded. Their haemorrhage locations were evaluated. Response to treatment and disease outcome data were recorded. Ethical approval for the study was obtained from the Ministry of Health and the Istanbul Prof. Dr. Cemil Taşçığlu City Hospital ethics board (Approval No.: 31 of 26.01.2021). The study conformed to the Helsinki Declaration.

Statistical Examinations: The NCSS (Number Cruncher Statistical System) (Kaysville, Utah, USA) software was used for statistical analyses. Complementary statistical methods (mean, standard deviation, median, frequency, ratio, minimum, maximum) were used when evaluating the study data. The suitability of quantitative data to normal distribution was tested using the Kolmogorov-Smirnov, Shapiro-Wilk test, and graphic evaluations. Student's t-test was used in twogroup comparisons of quantitative data presenting normal distribution, and the Mann-Whitney U test was used in twogroup comparisons of data without normal distribution. Pearson's Chi-square test was used in comparing qualitative data. Significance was considered at a minimum of $\mathrm{p}<0.05$.

\section{RESULTS}

Fifty patients with COVID-19-negative haemorrhage and 11 patients with COVID-19-positive haemorrhage receiving inpatient treatment in inpatient wards were recorded between March 11th, 2020, and March 11th, 2021. Among the patients who were COVID-19-negative, there were 17 women and 33 men, with a mean age of $62.88 \pm 8.78$ years. Among the patients who were COVID-19-positive, there were four women and seven men, with a mean age of $64.45 \pm 18.68$ years. The demographic data for patients who were COVID19-positive are presented in Table 1. There was no statistical difference in terms of age and sex between the two groups $(p=0.80)$. Haematologic data were evaluated in the two groups according to their distribution between the groups. No difference was found between the groups in terms of parametrically distributed PLT values $(p=0.377)$. The haemoglobin $(\mathrm{Hb})$ value was found to be statistically significantly lower in the COVID-19-positive group $(\mathrm{p}=0.012)$. No difference was found between the two groups in terms of the neutrophil, lymphocyte, leucocyte, INR, and neutrophile/lymphocyte ratios, which were seen to be distributed nonparametrically. The biochemical values and coagulopathy values of the COVID-19-positive cases are presented in (Tables 2 and 3).

The use of antiaggregants/anticoagulants was found to be significantly higher in the COVID-19-positive group $(\mathrm{p}=0.02)$. Twenty-two of 50 of the COVID-19-negative group were using antiaggregants and anticoagulants. Eleven patients were receiving ASA, six were receiving next-generation anticoagulants (NOAK), two had coumadin, and two patients were receiving dual treatments (ASA+clopidogrel, rivaroxaban+clopidogrel). Forty-two per cent of this group received treatment.

Seven of 11 of the COVID-19-positive group received antiaggregants and antiaggregants/low-molecular-weight heparin. In the term before hospitalisation, three were receiving acetylsalicylic acid (ASA), and one was receiving clopidogrel. This amounted to $36 \%$ of the patient group. The treatment of three patients was started at admission. The distribution of the medication taken by the patients is presented in Table 4.

Table 1. Comparison of demographic and hematologic data of COVID positive and negative cases

\begin{tabular}{lccc} 
& COVID-19 negative & COVID-19 positive & P value \\
& $\mathrm{n}=50$ & 11 & 0.804 \\
Age & $62.88 \pm 18.76$ & $64.45 \pm 18.68$ & 0.881 \\
Sex (Female/Male) & $17 / 33$ & $4 / 7$ & $\mathbf{0 . 0 0 2}$ \\
Drug abuse (-/+) & $28 / 22$ & $4 / 7$ & $\mathbf{0 . 0 2 7}$ \\
Survey (in life/exitus) & $36 / 14$ & $194.727 \pm 101635$ & 0.377 \\
Platelets & $224.000 \pm 70119$ & $10.2 \pm 3.2$ & $\mathbf{0 . 0 1 2}$ \\
Haemoglobin & $13.3 \pm 2.2$ & $878(144-2177)$ & 0.155 \\
WBC & $951(300-2866)$ & $697(149-2084)$ & 0.195 \\
Neutrophil & $723(29-2313)$ & $132(59-430)$ & 0.136 \\
Lymphocyte & $122(13-871)$ & $5.02(2.06-15.79)$ & 0.195 \\
Neutrophil/Lymphocyte ratio & $4.87(0.24-84.83)$ & $1.13(0.80-1.48)$ \\
INR & $1.09(0.87-4.94)$ & 0.180 & \\
\hline Whit blood &
\end{tabular}

WBC: White blood cell, INR: International Normalised Ratio 
Table 2. Hematologic values

\begin{tabular}{|c|c|c|c|c|c|c|c|c|c|}
\hline Case no. & $\begin{array}{l}\mathrm{WBC} \\
/ \mu \mathrm{L}\end{array}$ & $\begin{array}{c}\text { Neutrophil } \\
/ \mu \mathrm{L}\end{array}$ & $\begin{array}{c}\text { Lymphocyte } \\
/ \mu \mathrm{L}\end{array}$ & $\begin{array}{l}\mathrm{Hb} \\
\mathrm{g} / \mathrm{L}\end{array}$ & $\begin{array}{l}\text { PLT } \\
/ \mu L\end{array}$ & $\begin{array}{l}\text { D-dimer } \\
\text { ug/L }\end{array}$ & $\begin{array}{l}\text { CRP } \\
\mathrm{mg} / \mathrm{L}\end{array}$ & INR & aPTT \\
\hline Normal values & $3800-10,000$ & $1780-5380$ & $1320-3570$ & $130-175)$ & $150,000-400,000$ & $80-500$ & $<5$ & $0.8-1.2$ & \\
\hline 1 & 7360 & 6810 & 330 & 133 & 28,000 & 8120 & 223 & 1.13 & 24 \\
\hline 2 & 12,000 & 8910 & 430 & 108 & 143,000 & 1050 & 93 & 1.18 & 26 \\
\hline 3 & 1896 & 15,220 & 1520 & 113 & 289,000 & 4040 & 13.68 & 1.2 & 34.7 \\
\hline 4 & 8780 & 6230 & 820 & 130 & 202,000 & 680 & 91.1 & 1.01 & 24 \\
\hline 5 & 8250 & 6970 & 740 & 134 & 202,000 & 332 & 34 & 0.9 & 21.1 \\
\hline 6 & 11,000 & 10,320 & 1240 & 85 & 280,000 & 1480 & 101.6 & 1.48 & 31.3 \\
\hline 7 & 1440 & 1490 & 590 & 91 & 18,000 & 850 & 121.6 & 1.0 & 31.7 \\
\hline 8 & 21,770 & 2084 & 1320 & 62 & 350,000 & 1190 & 253.6 & 1.36 & 10.79 \\
\hline 9 & 7930 & 3520 & 700 & 107 & 195,000 & 2110 & 93 & 0.8 & 21 \\
\hline 10 & 6100 & 4820 & 1640 & 158 & 208,000 & 240 & 148 & 1.24 & 31.6 \\
\hline 11 & 14,560 & 12,610 & 1070 & 148 & 227,000 & - & 9.52 & 1.03 & 19.6 \\
\hline
\end{tabular}

Table 3. Coagulopathy values

\begin{tabular}{|c|c|c|c|c|c|c|c|c|}
\hline Case no & Ferritin & D- dimer & Procalcitonin & Fibrinogen & Urea & ALT & AST & $\mathrm{LDH}$ \\
\hline 1 & $*$ & 8120 & 37 & 523 & 41 & 73 & 93 & 432 \\
\hline 2 & 235.6 & 1050 & $*$ & $*$ & 117 & 69 & 82 & $*$ \\
\hline 3 & 1661 & 4040 & 0.5 & $*$ & 54 & 25 & 16 & 531 \\
\hline 4 & 257 & 0,68 & 0.72 & 483 & 19 & 14 & 33 & 188 \\
\hline 5 & 129 & 332 & 0.32 & $*$ & 23 & 94 & 37 & 745 \\
\hline 6 & 861.9 & 1.48 & 0.07 & 519 & 125 & 12 & 13 & 217 \\
\hline 7 & $*$ & 85 & 0,4 & 453 & 32 & 270 & 91 & 256 \\
\hline 8 & 4194 & 119 & 119 & 791 & 117 & 22 & 41 & 273 \\
\hline 9 & $*$ & 2110 & $*$ & $*$ & 72 & 10 & 17 & $*$ \\
\hline 10 & 2827 & 24 & 6 & 602 & 17 & $*$ & $*$ & 317 \\
\hline 11 & $*$ & $*$ & $*$ & $*$ & 31 & 17 & 24 & 306 \\
\hline
\end{tabular}

*No data

Table 4. Antiaggregant/anticoagulant distribution ratios by groups

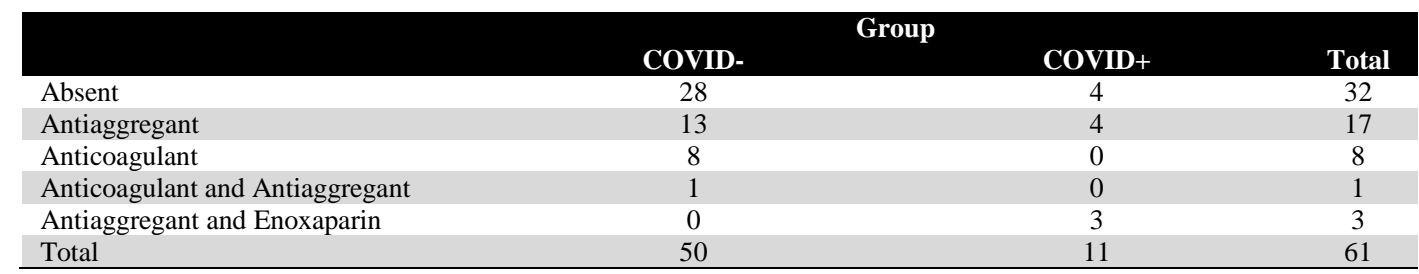

Table 5. Haemotoma location

\begin{tabular}{llccc} 
& & Group & & Total \\
\hline \multirow{5}{*}{ Hematoma location } & Putaminocapsular & 8 & COVID + & 9 \\
& Thalamic & 6 & 1 & 6 \\
& Pons & 5 & 1 & 6 \\
& Lobar & 14 & 6 & 20 \\
& SAH & 6 & 0 & 6 \\
& Subdural & 9 & 1 & 10 \\
\cline { 2 - 5 } Total & Infratentorial & 2 & 2 & 4 \\
\cline { 2 - 5 } & & 50 & 11 & 61 \\
\hline
\end{tabular}

SAH: subarachnoid hemorrhage

The most frequent comorbidity in the COVID-19-positive group was hypertension (HT) with $64 \%$, followed by diabetes mellitus (DM) with 45\%. Other comorbid diseases were ischemic heart disease, chronic obstructive pulmonary disease (COPD), chronic kidney failure, benign prostate hypertrophy, renal cell carcinoma, and glioblastoma multiforme. The first and second most frequent risk factors we identified in the COVID-19-negative patient group were HT (27/50) (54\%) and DM (11/50) (22\%). These were followed by concomitant coronary artery disease and trauma. Other risk factors were vascular malformation, polycythaemia vera, COPD, brain tumours, and cardiomyopathy.
There was no significant difference in terms of risk factors between the groups $(p=0.443)$. Although not statistically significant, vascular malformation and traumatic subarachnoid haemorrhage $(\mathrm{SAH})$ in the aetiology in the negative group were also noteworthy.

The haemorrhage distribution rates between the two groups are presented in the table. No difference was identified between the groups in terms of distribution location $(p=0.232)$ (Table 5). No difference was found between the groups in terms of clinical presentation $(\mathrm{p}=0.671)$ (Fig 1, brain CT images of 11 COVID-19-positive cases). 

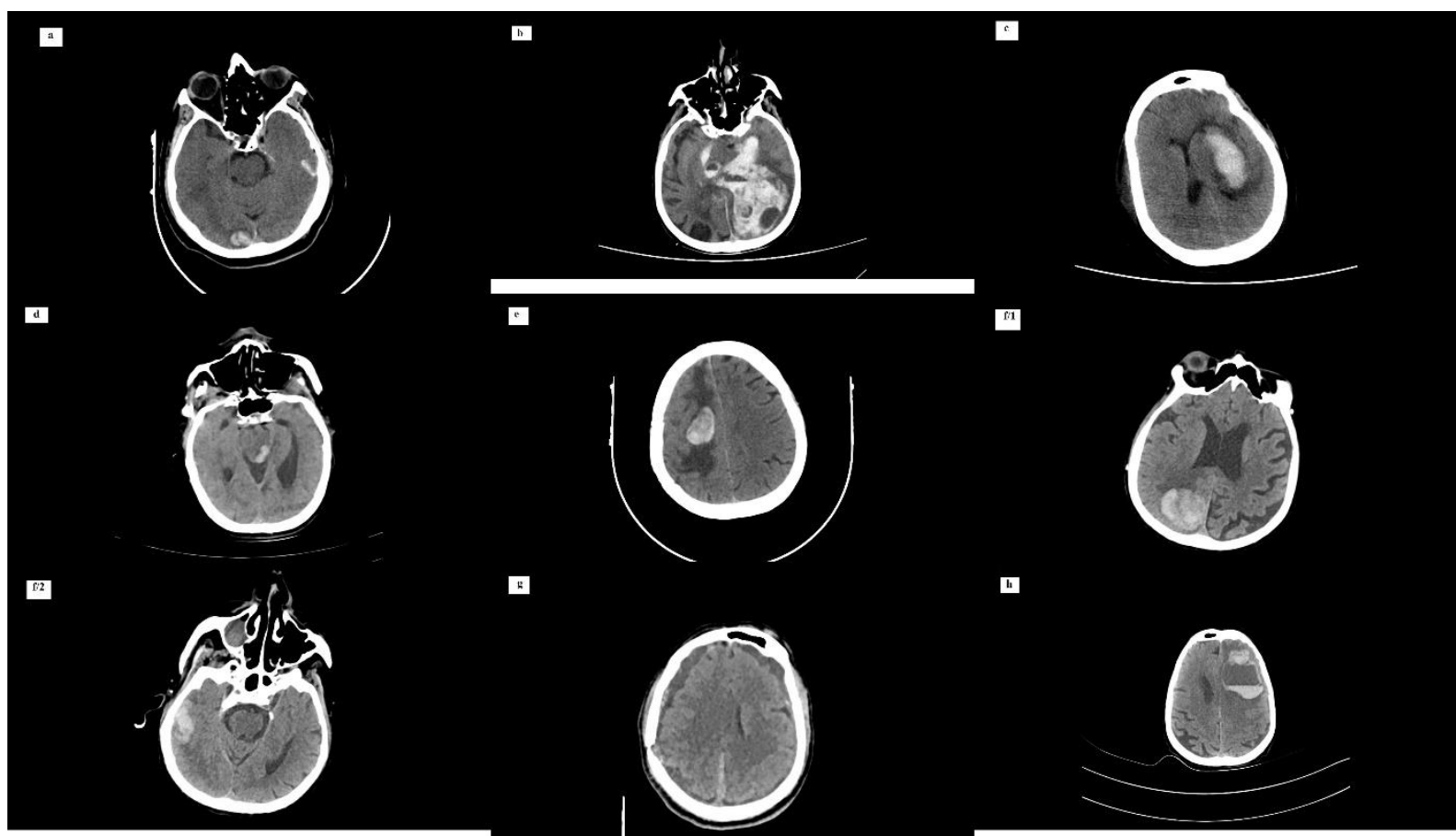

(11)

n

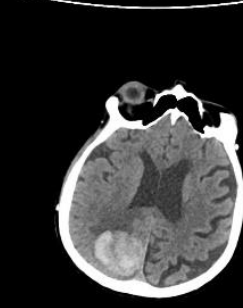

$\mathbf{4}$

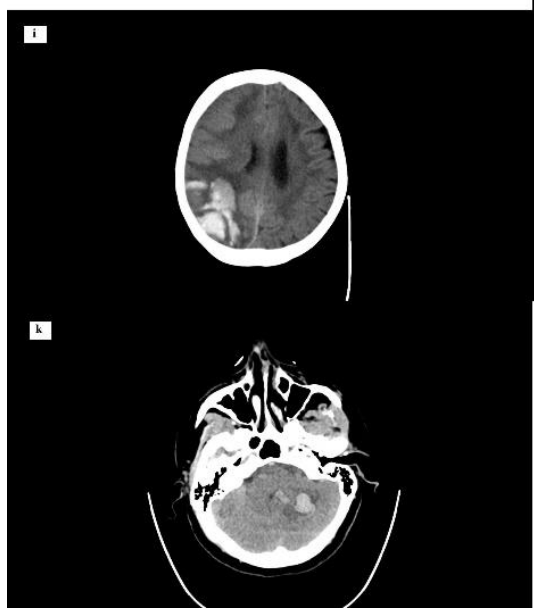

国
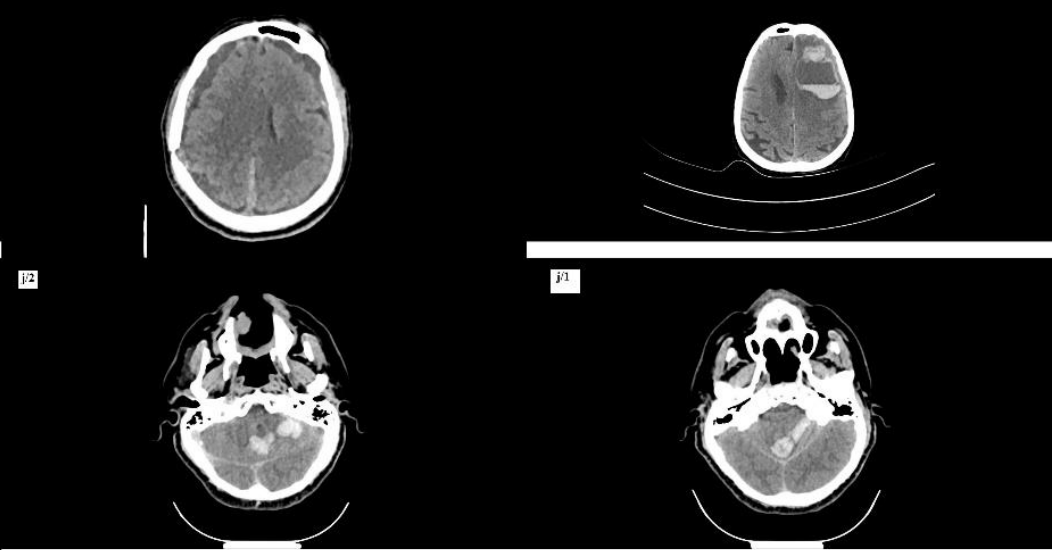

Fig 1: CT head for all COVID-19 patients. Cases 1-11, a-k, respectively.

The time between the diagnosis of patients with COVID-19 and the onset of haemorrhage varied between 0-15 days. Six of $11(55 \%)$ of our patients presented primarily with symptoms of haemorrhage, which was concurrent with the COVID-19 diagnosis. The haemorrhage time was day 0 of the presentation to the hospital in three patients using antiischemic treatment, and the 10th day in one patient.

A significant difference was found between the groups in terms of mortality rates $(p=0.024)$; the mortality rate was $64 \%$ in the COVID-19-positive group, whereas it was $28 \%$ in the negative group.

\section{DISCUSSION}

Haemorrhagic presentation concomitant with COVID-19 is infrequent but has serious consequences. In 1200 cases observed over 65 days, Pavlov et al. reported the rate of haemorrhage as $0.25 \%$ (5). In their study, Rothstein et al. reported the rate of ischemia as $2.4 \%$, and the rate of haemorrhage as $0-9 \%$ (6). Our rate was $0.12 \%$. We had a haemorrhage rate lower than that in the literature. We considered that this was due to our more precautious approach to administering antiaggregant treatment.
Our clinical approach involves administering anticoagulant doses mostly at prophylactic doses without rising to therapeutic doses. The sex distribution was consistent with that in the literature, where the majority of patients with COVID-19 with intracranial haemorrhage (ICH) were male $(65.8 \%)$ (3). Our cases also mostly consisted of men (64\%).

Although haemorrhage is seen less infrequently compared with ischemia in COVID-19, its mortality rate is higher. Severe pulmonary involvement also exacerbates negative outcomes. A mortality rate of $48.6 \%$ was reported in patients with COVID-19 with ICH. The mortality rate in our patients was found as $64 \%$. Therefore, it was somewhat high compared with mortality associated with haemorrhages due to other causes $(28 \%)$. Patients may present with a haemorrhage clinic without presenting pulmonary findings (7). This rate was $55 \%$ in our patients. Although COVID-19 is not associated with direct traumatic haemorrhages, dizziness and loss of balance associated with COVID-19 is the most reported neurologic finding, and traumatic secondary events may develop. During the pandemic, haemorrhages must be approached with caution considering that there may be an underlying COVID-19 infection, even if the aetiology is trauma. No SAH was seen in the study conducted by 
Altschula et al (7). No SAH was observed in our study either, and there were no patients with trauma in their aetiologies.

In our study, we evaluated major haemorrhages identified through brain CT scans. We did not include microhaemorrhages or minor haemorrhages developing from ischemia. Also, no magnetic resonance imaging scans were taken for these diagnoses in these patients (8). Multifocal and multicompartmental ICHs were observed concomitant with more severe COVID-19 findings. These were associated with multiple organ failure, disseminated intravascular coagulation, and iatrogenic anticoagulant use. The risk of haemorrhage must be considered when prescribing anticoagulants in treatment regimens (9). Dogra et al. presented 33 patients with $\mathrm{ICH}$, all of whom were receiving anticoagulants at therapeutic doses (9). Only four of our patients were receiving antiaggregant during their inpatient treatment, and no oral anticoagulants were administered. This may be interpreted as an illustration of the contribution of the vascular impacts of COVID. Patients developing haemorrhages in the course of the COVID-19 treatments after admission were receiving enoxaparin sodium 4000 IU, 6000 IU or $8000 \mathrm{IU}$ in addition to ASA $100 \mathrm{mg}$. It was observed that haemorrhage appeared on the 8-15th days of this treatment. The most frequent comorbidity was HT. The second most frequent was DM. This distribution was similar to haemorrhages not concomitant with COVID-19. This led us to believe that greater care should be taken in the dosages and durations in administering antithrombotics/antiaggregants to patients with diabetes with a high risk of haemorrhage, especially those who have high blood pressure, or unregulated arterial pressure and blood sugar.

In a case reported in the literature, a patient receiving a combination of ASA and clopidogrel was administered a therapeutic dose of enoxaparin. This caused the development of a haemorrhage that resulted in mortality in the patient. The coadministration of anticoagulant treatment in those receiving antiaggregants must be carefully considered. A brain CT scan must be obtained before beginning the triple treatment, and proper blood pressure control must be ensured (10). Very severe ICH resulting in brain death was observed in three patients in whom anticoagulants were used in therapeutic doses. All cases resulted in brain death (11).

Also, during the pandemic, arteriovenous malformation, cavernous malformation, dural arteriovenous fistula, cerebral sinus tumours, and brain tumours that could be secondary ICH causes, should not be ignored. Not all haemorrhages that are observed are COVID-19-related (12). Like everywhere in the world, elective surgery could not be performed in Turkey during the pandemic. Many surgeries were postponed due to the shortage of beds in intensive care units (ICUs). The data we reported were evaluated from patients brought to the hospital. Work was performed under extraordinary circumstances during the pandemic. Along with minor cases that did not reach the hospital due to the pandemic, we also believe that there were deaths outside the hospital associated with severe neurologic findings.

Post-mortem brain studies have also shown direct invasion of neurons and glial cells (13). It was emphasised that D-dimer levels were five times higher in $\mathrm{ICH}$ in hospitalized patients with COVID-19 (14). In $73 \%$ of our patients, D-dimer was found to be high in the range of 1.5-8 times. The necessity to evaluate the risks and benefits of an anticoagulant treatment regimen has been emphasised in a series of six cases in which haemorrhage developed under intensive care. It is also indicated that a change in the neurologic condition may be noticed belatedly in such patients due to reasons such as isolation and sedation (14).

Wang et al. showed an association of a high neutrophillymphocyte ratio (NLR) with 30-day mortality in patients with ICH and various studies that indicated that NLR might be an independent predictor of ICH outcomes (15). COVID19 produces an inflammatory cascade, and a higher NLR at hospital admission has been associated with a more severe outcome (16). In our patient group, in six of the seven cases ending in mortality, NLR was found to be 7.5-20 times higher. However, this rate was not statistically different from the COVID-19-negative cases. Among the haematologic parameters that were evaluated, only the $\mathrm{Hb}$ value was found to be significantly lower in the positive group. However, the correlation of this low value with the survey was not identified.

COVID-19 has been documented to enter vascular endothelium, leading to endotheliitis, which could trigger the microthrombosis of small penetrating arteries, and lead to an increased risk for ICH (17-18). In one retrospective study, thrombocytopenia with platelet counts of $<150,000 / \mathrm{L}$ and elevations in D-dimer of $>2500 \mathrm{ng} / \mathrm{mL}$ at initial presentation were also predictive of bleeding complications during hospitalisation (19). In our patients, bleeding was seen even without such high D-dimer values. Only two of our patients presented an outcome above that value. Early-stage COVID19 CRP levels are known to positively correlate with lung involvement and may reflect disease severity (20). The CRP values of all our patients were high, and involvement was present in thoracic CT in all patients.

Some mechanisms can be considered in the tendency of these patients to develop subdural haemorrhage SDH. The point of entry for COVID-19 into human tissue is mediated primarily by a specific cellular receptor, angiotensin-converting enzyme 2 (ACE-2). In our series, subdural haemorrhage was seen in one case. With respect to bleeding distribution rates in a study evaluating haemorrhages, single compartments were involved in the rest, with intraparenchymal haemorrhage (IPH) being the most common variety $(62.6 \%)$, followed by SAH $(15.0 \%), \mathrm{SDH}(11.6 \%)$, and intraventricular haemorrhage (IVH) (1.4\%) (3). In a study evaluating 18 patients, proof of acute intracranial bleeding was found within 11 days following presentation (IQR: 9-29). Six (33.3\%) patients presented with parenchymal bleeding, 11 (61.1\%) presented with $\mathrm{SAH}$, and one $(5.6 \%)$ patient presented with subdural bleeding. Three patients presented with IVH (16.7\%) (21). In addition to there being limited data in the literature, different results have also been reported in terms of haemorrhage location. Except for one, all 11 of our cases had intraparenchymal bleeding.

The limitation of our study is that it is a retrospective, crosssectional, and single-centre study. The patients included in the study had major bleeding identified through CT scans. No magnetic resonance scanning was performed, and no incidence study was performed from among all patients with COVID-19. 


\section{CONCLUSIONS}

Although less frequent compared with ischemic cerebrovascular events, it is important to identify ICHs because they present a higher mortality rate. Independent of the use of antiaggregants, the presenting symptom in patients with COVID-19 may be haemorrhage, and the haemorrhage and COVID-19 diagnoses may be concurrent. Caution must also be exercised concerning haemorrhage in inpatients using anticoagulants at a prophylactic dose. Neurologic examination findings must be monitored carefully in clinics and ICUs, and the risks to which the patients are subjected concerning the choice and doses of antiaggregant/anticoagulant treatment must be evaluated; the risk/benefit ratio must be carefully considered.

Author Contributions: SÜÖ, CE: Research of the literature, Patient examinations, Manuscript preparation and revisions.

Financial \& competing interest's disclosure: The authors have no relevant affiliations or financial involvement with any organisation or entity with a financial interest in or financial conflict with the subject matter or materials discussed in the manuscript. This includes employment, consultancies, honoraria, stock ownership or options, expert testimony, grants or patents received or pending, or royalties.

Conflict of interest: The author declared no potential conflicts of interest with respect to the research, authorship, and/or publication of this article. This research did not receive and specific grant from funding agencies in the public, commercial, or not-for-profit sectors.

\section{REFERENCES}

1. Zhou F, Yu T, Du R, et al. Clinical course and risk factors for mortality of adult inpatients with COVID-19 in Wuhan, China: a retrospective cohort study. Lancet 2020;395:1054-1062.

2. Wang T, Chen R, Liu C, et al. Attention should be paid to venous thromboembolism prophylaxis in the management of COVID-19. Lancet Haematol 2020;7:e362-e363

3. Cheruiyot I, Sehmi P, Ominde B, Bundi P, Mislani M, Ngure B, Olabu $\mathrm{B}$, Ogeng'o JA. Intracranial hemorrhage in coronavirus disease 2019 (COVID-19) patients. Neurol Sci 2021; 42:25-33

4. Connors JM, Levy JH. COVID-19 and its implications for thrombosis and anticoagulation. Blood 2020;135 (23): 2033-2040.

5. Pavlov V, Beylerli O, Gareev I, Torres Solis LF, Solís Herrera A and Aliev G. COVID-19-related intracerebral hemorrhage. Front. Aging Neurosci.2020; 12:600172. doi: 10.3389/fnagi.2020.600172

6. Rothstein A, Oldridge O, Schwennesen H, Do D, Cucchiara BL, Acute Cerebrovascular Events in Hospitalized COVID-19 Patients. Stroke. 2020;51:e219-e222.
7. Altschula DJ, Undaa SR, Garza Ramosa RL, Zampolinb R, Bentona J, Hollanda R, Fortunela A, Haranhallia N. Hemorrhagic presentations of COVID-19: Risk factors for mortality Clinical Neurology and Neurosurgery 2020;198 106112

8. Radmanesh A, Derman A, Lui YW, Raz E, Loh JP, Hagiwara M, Borja MJ, Zan E, Fatterpekar GM, COVID-19-associated Diffuse Leukoencephalopathy and Microhemorrhages. Radiology 2020; 297:E223-E227

9. Dogra S, Jain R, Cao M, Bilaloglu S, Zagzag D, Hochman S, Lewis A Melmed K, Hochman K, Horwitz L, Galetta S, Berger J, Hemorrhagic stroke and anticoagulation in COVID-19Journal of Stroke and Cerebrovascular Diseases,2020; 29(8 ) 104984

10. Bhanu Gogia, Xiang Fang, Prashant Rai Intracranial Hemorrhage in Patient With COVID-19: Possible Explanations and Considerations cureus 2020; 12(8): e10159. DOI 10.7759/cureus.10159

11. Ghani MU, Kumar M, Ghani U, Sonia F, Abbas SA. Intracranial hemorrhage complicating anticoagulant prophylactic therapy in three hospitalized COVID-19 patients Journal of NeuroVirology 2020; 26:602-604

12. Montemurroa N. Intracranial hemorrhage and COVID-19, but please do not forget "old diseases" and elective surgery Brain, Behavior, and Immunity 2021;92 207-208

13. Aghagoli G, Gallo Marin B, Katchur NJ, Chaves-Sell F, Asaad WF, Murphy SA. Neurological involvement in COVID-19 and potential mechanisms: a review. Neurocrit Care 2020.

14. Fady Mousa-Ibrahim FM, Berg S, Od`TPDetola O, Teitcher M, Ruland S, Intracranial Hemorrhage in Hospitalized SARS-CoV-2 Patients: A Case Series Journal of Stroke and Cerebrovascular Diseases 2021:30 (1) 105428

15. Wang F, Wang L, Jiang TT, Xia JJ, Xu F, Shen LJ, et al. Neutrophil tolymphocyte ratio is an independent predictor of 30-day mortality of intracerebral hemorrhage patients: a validation cohort study. Neurotox Res 2018 .

16. Ciccullo A, Borghetti A, Zileri Dal Verme L, et al. Neutrophil- tolymphocyte ratio and clinical outcome in COVID- 19: a report from the Italian front line. Int J Antimicrob Agents 2020;56(2):106017.

17. Moriguchi T, Harii N, Goto J, et al. A first case of meningitis/ encephalitis associated with SARS-Coronavirus-2. Int J Infect Dis 2020;94:55-58.

18. Varga Z, Flammer AJ, Steiger P, et al. Endothelial cell infection and endotheliitis in COVID-19. Lancet 2020;395 (10234):1417-1418.

19. Al-Samkari H, Karp Leaf RS, Dzik WH, et al. COVID-19 and coagulation: bleeding and thrombotic manifestations of SARS-CoV-2 infection. Blood 2020;136(4):489-500. Al-Samkari H, Karp Leaf RS, Dzik WH, et al. COVID-19 and coagulation: bleeding and thrombotic manifestations of SARS-CoV-2 infection. Blood 2020;136(4):489-500.

20. Wang, L. C-reactive protein levels in the early stage of COVID- 19. Med. Mal. Infect.2020 50, 332-334.

21. Nawabi J, Morotti A, Wildgruber M, Boulouis G, Kraehling H, Schlunk $\mathrm{F}$ et al. Clinical and imaging characteristics in patients with SARSCoV-2 infection and acute intracranial hemorrhage. J Clin Med 2020 $9(8): 2543$ 